Anuario del Instituto de Historia Argentina, vol. 17, n 2, e046, diciembre 2017.

ISSN 2314-257X

Universidad Nacional de La Plata.

Facultad de Humanidades y Ciencias de la Educación.

Centro de Historia Argentina y Americana

\title{
De los Habsburgo a los Borbones en el Rio de la Plata. A trescientos años del gobierno de Bruno Mauricio de Zavala
}

\author{
Griselda Tarragó *; Carlos María Birocco ** \\ * Centro de Investigaciones de la Facultad de Filosofía y Humanidades (CIFFyH) \\ Universidad Nacional de Córdoba; ** Centro de Historia Argentina y Americana (CHAyA) \\ Universidad Nacional de La Plata, Argentina | griseldatarrago@hotmail.com; \\ cmbirocco@hotmail.com
}

Según algunos testimonios, Bruno Mauricio de Zavala fue pocas veces hasta la casa capitular de Buenos Aires. Ataviado con sus suntuosos ropajes y con toda la distinción que su cargo político y su rango militar ameritaban, sus contemporáneos lo describen como un hombre con presencia que inspiraba temor: su brazo de plata que suplía al que la guerra se había llevado en Lérida defendiendo el reinado de Felipe V, era quizá uno de sus mayores atributos alegóricos de su carrera de honores en el servicio al rey. Su ausencia de la casa consistorial no se atenía a un capricho o a una coyuntural mala relación con los hombres del ayuntamiento. Él había llegado para validar y concretar la voluntad real en ese recóndito e indómito extremo sur del virreinato peruano. Las voces discordantes que se elevan, las oposiciones del cabildo, las dificultades con las que se topa permiten construir una pintura compleja de esta sociedad, con la multiplicidad de cuerpos que ponían en ejercicio sus antiguos derechos jurisdiccionales y de las dificultades que encuentra el ordenamiento borbónico para meter cuña, aún con un gobernador fiel y la potestad real puesta a su disposición.

En la observación de la complicada gestión de este gobernador, al que se reconoce como el primero puramente "borbónico", se cruzan inexorablemente diferentes miradas teóricas y metodológicas de molde disímil. Esas miradas se entretejen en la observación de la vitalidad que la Monarquía compuesta -en cuanto cultura política- tiene en pleno siglo XVIII, como en la presencia inexorable 
de agencias y redes que configuran y le dan sentido, tanto a las formas de ejercicio del poder como a la ordenación misma de esa sociedad bajo unas consignas del soberano que mandaban la faena de una nueva territorialidad desde las entrañas mismas de sus propias tensiones y contradicciones.

El quiebre del 1700 sería para la Monarquía española un año signado no sólo por el traspase temporal de un siglo que concluía. La larga Guerra de Sucesión que allanaría el espinoso camino de la casa de Borbón al trono, expandió y densificó los conflictos europeos en todos los territorios hispánicos. América en general, y algunos puntos álgidos como la cuenca rioplatense y las costas en Suramérica, variaron en su calidad estratégica hacia una centralidad antes no experimentada. En ese contexto, se propone esencialmente pensar el problema de esa reconfiguración espacial en clave política, territorial, buceando en el tema de las llamadas “reformas borbónicas”, pero en un periodo temprano y todavía poco definido en lo institucional.

Las coyunturas de una cruenta guerra, las necesidades de un rey necesitado de recursos y apoyos políticos, los avances de los enemigos sobre puntos del mundo americano antes menos amenazados obligaron a la Corona a generar cambios de estrategias políticas. Nuevos hombres, reformas institucionales y diferentes cursos de acción coadyuvaron para que la gobernación de Buenos Aires comenzara su lenta transformación, donde también se habían comenzado a manifestar los indicios de un proceso de reorientación atlántica ya en el siglo XVII. Limitado en sus alcances espaciales y económicos al nacer la centuria, se hizo contundente e irreversible desde la segunda mitad del siglo XVIII.

Interesa entonces investigar las motivaciones que condujeron al rey a tomar una serie de decisiones que permitieran transferir mayores y diferentes recursos a ese territorio hasta entonces marginal o fronterizo para la atención regia. Al mismo tiempo, cómo esa situación se producía por agencias convergentes (la monárquica, pero también la de cuerpos y agentes diversos) un nuevo equipamiento político del territorio.

La historiografía ha contrastado con frecuencia las políticas de los últimos Habsburgo con las de los Borbones. Los últimos dos monarcas de la dinastía saliente se habrían valido de la venalidad para la cobertura de cargos como los de gobernador u oidor, mientras que la nueva familia reinante renunció gradualmente a esa práctica para concentrarse en la formación de un estamento de servidores fieles y eficientes en la función del gobierno. En el caso del Río de la Plata, el primer paso consistió en reforzar los controles ejercidos desde la península al destinar el gobierno de esta plaza a militares de carrera.

Una guerra mucho más “mundializada” estableció los parámetros de una nueva geoestrategia. Ya no se trataba de conservar los dominios en el Río de la Plata, en el sentido que había tenido hasta entonces. Había que pasar a la ofensiva frente al avance inglés y portugués porque las razones profundas de conservar-mantener este territorio seguían vigentes: la trastienda de Potosí se hallaba ahora en un peligro más certero y amplificado, especialmente desde la implantación de la colonia de Sacramento en 1680, frente a la misma Buenos Aires. Los nuevos objetivos que despuntan en la gestión de estos territorios buscaban reforzar su control a través de un potenciado aparato militar y un aumento relativo de los recursos que se destinaban al mismo.

El arribo del gobernador Bruno Mauricio de Zavala representa el inicio de un periodo esencialmente 
“borbónico”. Emergente destacado del proceso socio-político que elevó a élites vascas y navarras a las altas esferas de la política, agenció una importante retahíla de medidas que permitieron poner en marcha el proceso de transformaciones en los planos administrativo, hacendístico y militar que se consolidarían en el último cuarto del siglo. Nacido en Durango de Vizcaya, había iniciado sus servicios a la corona en el ejército de Flandes y formó parte de un destacado grupo de hombres de armas que habían demostrado su probada fidelidad a la causa borbónica y se habían destacado por sus carreras exitosas y por su participación en las empresas bélicas del rey.

Al destinarlo al Río de la Plata, Felipe V atendía a la necesidad de colocar un hombre fuerte en una región cuyo manejo había resultado problemático para la corona. En Buenos Aires, ciudad cabecera, Zavala debió poner límites a grupos de poder tradicionalmente implicados en el contrabando y proclives a la lucha facciosa. Tuvo, además, un decidido protagonismo en la configuración territorial de dicha gobernación, interviniendo activamente en tres de sus franjas fronterizas, que necesitaban ser aseguradas: la del norte de Santa Fe frente al embate constante de grupos chaqueños, la del río Salado del sur frente a las incursiones de los araucanos, y la de la Banda Oriental, donde debió enfrentarse con las pretensiones expansionistas de los portugueses.

Estas líneas historiográficas y conceptuales son las que nos han impulsado al convocar a este Dossier. Por tal motivo también, nos hemos permitido pensar el periodo en un proceso mucho más extenso, que excede la gobernación de Zavala: sabido es el valor estratégico que otorgó la Monarquía a la Gobernación del Río de la Plata desde el reinado de los últimos Habsburgo, que la convirtió en el principal bastión militar de la frontera sur del extenso Virreinato del Perú. Analizar y comprender su gestión en un recorte de larga duración nos permite explorar y -al mismo tiempocuestionar, romper con superados rompimientos anclados en criterios seculares o estrictamente dinásticos.

El primer artículo del Dossier es el de Oscar Trujillo. Se propone ofrecernos un perfil de los gobernadores del Río de la Plata a lo largo del siglo XVII, brindándonos en paralelo algunos lineamientos sobre el proceso de dotación militar de este enclave tan lejano y marginal del imperio español. Considera este autor que la corona tuvo muy en cuenta las atribuciones militares al seleccionar a los hombres que ocuparon ese cargo. Ha hallado que estos no fueron solamente oficiales escogidos por haberse distinguido en las múltiples campañas bélicas llevadas a cabo por la corona tanto en frentes europeos como africanos o americanos, sino también por haber sido la mayor parte de ellos aceptados por una orden militar como la de los Caballeros de Santiago. Esa pericia en las armas, ya en la segunda mitad de ese siglo, se juzgó necesaria para atender dos frentes: la creciente presencia de indios pampas y serranos en las llanuras bonaerenses y el asentamiento de los portugueses en la Colonia do Sacramento.

Trujillo ha hallado, empero, una visible contradicción entre la destacada formación militar de los gobernadores y el pobre equipamiento de las fortificaciones de Buenos Aires. A lo largo del siglo XVII, el compromiso cada vez mayor en destinar a esta plaza recursos humanos desde la península -gobernadores militares, oficiales experimentados, partidas de soldados- se contrapuso con las pobres inversiones que se hicieron en Real Fortaleza de San Juan Baltasar de Austria, que era el único baluarte defensivo de esta ciudad. En su correspondencia con el Consejo de Indias, los gobernadores no dejaron de advertirle sobre las amenazas del asedio de una armada enemiga o el 
ataque de corsarios y a sugerirles que se planificara una mejora en las condiciones de este emplazamiento, pero a lo largo de esa centuria no conseguirían casi ningún avance al respecto. Resulta destacable el esmero puesto por dos gobernadores, Joseph Martínez de Salazar y Andrés de Robles, en informar a la Junta de Guerra de dicho Consejo sobre las debilidades del sistema defensivo y proponer reformas. Aunque sus propuestas y advertencias tuvieron escaso eco, sus informes permiten reconocer en ellos un acabado conocimiento tanto de las tácticas defensivas como del territorio.

El segundo artículo del Dossier es el de Carlos Birocco. Este se enfoca en el período inmediatamente posterior al de Trujillo: el del recambio dinástico, es decir, el período de transición que se prolongó entre la última década del siglo XVII y la tercera del siglo XVIII. Su propuesta es analizar los vínculos entre los gobernadores y la vecindad de Buenos Aires. Aquellos mostraron una visible avidez por enriquecerse a expensas del cargo a través de la corrupción, la extorción y el contrabando; ello los condujo a sostener una estrecha alianza con un sector muy acotado de vecinos, que pondrían a su disposición los conocimientos que tenían sobre el entorno local a cambio de recibir una participación en esos beneficios. Al favorecer a unos y excluir a otros, se alentarían el faccionalismo y los enfrentamientos en el seno del cabildo, siendo esta corporación el lugar donde alcanzaron su mejor expresión esas disputas.

Esta coyuntura política se modificaría en la segunda década del siglo XVIII gracias a la intervención del monarca, que anteriormente se había mantenido al margen debido a hallarse insumido en la Guerra de Sucesión. Envió a primero a un pesquisidor, Juan Joseph de Mutiloa y Andueza, a punir los excesos del gobernador Velasco, que había contrabandeado con los franceses, y luego modificó la estructura jerárquica local, creando nuevos funcionarios que en adelante contrabalancearían el poder de los gobernadores, como serían el teniente de rey y el veedor del presidio. Un último paso en este primer ciclo de reformas sería la colocación de gobernadores militares que habían ofrecido su lealtad y sus servicios a la nueva dinastía, como lo fueron Baltasar García Ros y Bruno Mauricio de Zavala. Este último pondría su energía en moderar a la dirigencia del cabildo de Buenos Aires y combatiría al faccionalismo, al punto de purgar a dicho ayuntamientos de los miembros que manifestaron oponérsele.

Tanto Trujillo como Birocco juegan en sus artículos con un simbolismo que recogen en las fuentes documentales: el de considerar al puerto de Buenos Aires como una "llave”. Para los Habsburgo, esa llave había consistido en nombrar a destacados militares como gobernadores de esta plaza y acumular en ellos poderes y facultades, tolerando incluso la apropiación de los que no le estaban explícitamente destinados. A lo largo del siglo XVII, el cabildo y los oficiales de la Real Hacienda se quejarían de los excesos que generaría dicha acumulación. Entre fines de esa centuria y comienzos de la siguiente, la exacerbación de esos poderes llevó a la Monarquía a revisarlos y, sobre todo, a sopesarlos y delimitarlos. Esa era la nueva concepción de la llave que impondrá el primero de los Borbones.

En tercer lugar en el Dossier se encuentra el artículo de Rafael Guerrero Elecalde, quien analiza la figura de Bruno Mauricio de Zavala como representante de uno de los colectivos periféricos que habrían de ser privilegiados por su fidelidad a la causa borbónica durante la Guerra de Sucesión: los llamados "norteños", vascos y navarros. Estas familias, enlazadas por medio de alianzas 
matrimoniales y parentales, eran dueñas de cuantiosas fortunas procedentes de la producción de hierro, del comercio en los puertos del Cantábrico y de sus negocios con la corona. Ya habían ingresado en la alta administración y el ejército durante los últimos Habsburgo, pero el apoyo incondicional que brindaron a la nueva dinastía produjo su afianzamiento y los catapultó a los espacios de poder más significativos en el seno de la Monarquía, quedando desde esa posición ligados a las transformaciones en el aparato administrativo, hacendístico y militar que impulsó Felipe V.

Guerrero Elecalde considera a Zavala representativo de ese colectivo, tanto por sus inversiones y sus actividades económicas como por la manera en que fue promovido en la administración imperial gracias al apoyo de las redes familiares y paisanales. Los inicios de su carrera estuvieron ligados a la remodelación del ejército que impuso dicho monarca, quien sustituyó los antiguos cuerpos cortesanos por cuerpos de elite y tropas de primera línea en el combate. En ese contexto de transformación, Zavala acompañaría a Flandes a uno de sus paisanos, Juan de Idiáquez, que apadrinaría su ascenso al grado de capitán. Su formación militar favorecería su ingreso en la planta funcionarial, que debería mucho a la gestión de los miembros de otra parentela norteña, los Villarreal de Bérriz. Estos, que ocupaban un lugar destacado en el entorno de Felipe V, eran sus primos y seguramente incidieron en la decisión del monarca de designarlo en 1717 gobernador del Río de la Plata. Una década más tarde, enterados de la arrolladora labor que se le encomendó aquí, marcada por las campañas militares en múltiples frentes, intentaron nuevamente influir para que se le diera otro destino menos problemático y consiguieron que lo nombraran presidente de la Capitanía General de Chile. Aunque en 1736 la muerte impidió a Zavala ser investido en ese nuevo cargo, su promoción formó parte de una política familiar que movilizó a los integrantes de una “casa” para situarlos en los espacios y lugares adecuados donde podrían ser útiles para llevar adelante diversas operaciones que podían resultarles beneficiosas.

El artículo siguiente del Dossier, escrito por Griselda Tarragó, complementa y profundiza los aportes hechos por Guerrero Elecalde al análisis de la figura de Bruno Mauricio de Zavala, y le agrega a la misma una dimensión más “americana”. Esta autora cuestiona las limitaciones de la categoría historiográfica de “reformismo borbónico" si se la vincula solamente con las transformaciones que llevó a cabo Carlos III, y plantea que en el Río de la Plata los cambios en la administración política comienzan durante la segunda década del reinado de Felipe V. Este monarca, ciertamente, reorganizó la administración del reino sin modificar sus bases teóricas, pero marginó a sectores tradicionalmente poderosos como la aristocracia castellana y eligió nuevos hombres para que lo secundaran, que aunque se hallaban desprovistos de peso político propio habían manifestado ser leales al monarca. Entre ellos se hallaría Zavala, que habría de encarnar las nuevas estrategias de la corona en relación al Río de la Plata.

Zavala llegó a Buenos Aires luego de que esta ciudad experimentara el enfrentamiento entre facciones, y en momentos en que los portugueses se habían reposicionado en Colonia do Sacramento y amenazaban con apropiarse de la bahía de Montevideo. En este contexto se explica que se confiara el mando a un militar calificado como él, en momentos en que la militarización en la función de gobierno se apreciaba en los territorios de la Monarquía a ambos lados del océano. Lo que caracterizaría su larga gestión, explica Tarragó, sería la simultaneidad de conflictividades 
bélicas y socio-políticas altamente violentas que debería enfrentar. Todo ello se vería complicado por la falta de recursos con que solventar el plan de acción que le encomendaron sus superiores, primero para enfrentar a los portugueses en la Banda Oriental y luego a la rebelión de los comuneros en Asunción. Por tal razón debería recurrir al auxilio que le facilitó su trama relacional: por ejemplo, a los militares vizcaínos que lo auxiliaron en Buenos Aires y otras ciudades de la gobernación, quienes conformaban una sólida red que mantenía el orden durante su ausencia, o a los padres jesuitas, que le suministraron los ejércitos indígenas para emprender sus campañas.

El quinto y último artículo del Dossier es el de Pablo Fucé, que sitúa la fundación de la ciudad de Montevideo como obra de la gestión de Bruno Mauricio de Zavala. Ya en las primeras décadas del siglo XVII, como lo expresa el artículo de Trujillo, el gobernador Céspedes advertía al Consejo de Indias sobre el riesgo de que las potencias enemigas se apoderaran del puerto de Montevideo, y le recomendaba "hacer población” allí. Pero no fue hasta 1723 en que el monarca portugués Juan V ordenaría la ocupación efectiva de dicho puerto. Siguiendo sus instrucciones, el maestre de campo Manuel Freitas da Fonseca comandó una expedición de 250 hombres que tomó posesión del mismo, pero en forma inmediata Zavala cruzó el estuario para asediarlo y lograr su retirada. Poco más tarde, este último ordenó al ingeniero Domingo Petrarca diseñar la fortificación de la nueva ciudad y su amanzanamiento. El proceso fundacional se completaría con el reparto de solares y chacras entre los pobladores entre 1727 y 1728 y con la instalación del cabildo y la construcción de la primera iglesia matriz en 1730 .

Paralelamente, observa Fucé, se fijaron las celebraciones de la ciudad, que conformaron la matriz de la trama simbólica litúrgica y ceremonial de autoridades y pobladores, poniéndose la misma bajo el patronato de los Santos Felipe y Santiago. Las relaciones con las parcialidades indígenas inmediatas, particularmente con los minuanes, fueron desde el comienzo conflictivas, pero en 1732 Zabala celebró con ellos una ceremonia de pacificación que, de acuerdo con este autor, inició un periodo de tensa calma que se prolongaría hasta mediados de siglo. Otra situación conflictiva fue la relación entre el cabildo montevideano y los comandantes enviados desde Buenos Aires, que aunque formalmente carecían de funciones políticas se excedieron en sus competencias y al juzgarse responsables del orden político participaban de las deliberaciones del ayuntamiento. No obstante, los miembros de la dirigencia capitular supieron desempeñarse como oficiales de la Monarquía, manifestando obediencia y lealtad a sus superiores y eficiencia en la administración, y aprendieron además a negociar como corporación, al punto de conseguir que en 1744 uno de sus alcaldes ordinarios se recibiera del cargo de teniente de rey de aquella plaza.

Muchos años después de esta gobernación emblemática , la creación del Virreinato del Río de la Plata en 1776 plasmó no sólo la apertura oficial y definitiva de este espacio hacia la Metrópoli, sino también la introducción del mismo en el gran proceso denominado de "reformas borbónicas", durante el reinado de Carlos III. Entendemos que esta sanción, en esta época tan tardía, expresa la conclusión de un largo proceso que se remonta al siglo anterior, más que el momento fundacional de una nueva etapa. La transferencia de centralidad desde un denominado "espacio peruano" a un "espacio atlántico" fue la resultante de la formación de un candente magma alimentado por las tensiones que comenzaron a manifestarse entre múltiples actores (entre los cuales la corona no fue sino uno más) en un momento en que las disputas por el equilibrio europeo se tornaron particularmente álgidas y revirtieron en conflictos sin precedentes por los espacios coloniales, los cuales comenzaban a adquirir una nueva dimensión para la Corona española, 
especialmente por los avances de sus representantes sobre un renovado ideal de monarquía que intentaba no sólo generar un modelo más eficiente de ejercicio del poder, sino también un control más eficaz y centralizado de su administración. De esos procesos y tiempo específico trata fundamentalmente este dossier... 